\section{Kompass \\ Autoimmun}

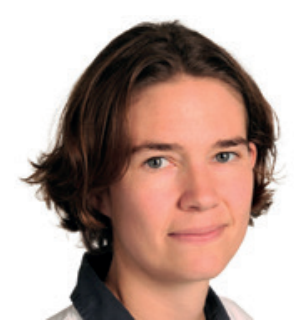

\section{Katharina Warncke}

Klinik für Kinder- und Jugendmedizin, München Klinik Schwabing und Klinikum rechts der Isar der Technischen Universität München, München, Deutschland

Institut für Diabetesforschung, Helmholtz Zentrum München, Deutsches Forschungszentrum für Gesundheit und Umwelt, München-Neuherberg, München, Deutschland

\title{
Früherkennung und Immunintervention: Neue Wege bei Typ-1-Diabetes
}

Sehr geehrte Leserinnen und Leser, Ich freue mich sehr, Sie heute zur Lektüre der neuen Ausgabe von Kompass AuTOIMmuN begrüßen zu dürfen. Wie Sie wissen, sind Autoimmunerkrankungen auf dem Vormarsch - und machen leider auch vor Kindern und Jugendlichen nicht halt. Dieses Heft beschäftigt sich unter anderem mit dem Thema «Typ-1-Diabetes». In den letzten Jahrzehnten zeigt sich ein deutlicher Anstieg der Inzidenz, im letzten Jahr zudem - vermutlich durch die Pandemie und den dadurch erschwerten Zugang zur Medizin für so manche Familie - auch ein dramatischer Anstieg von diabetischen Ketoazidosen bei Manifestation.

Während früher die klinische Manifestation von Typ-1-Diabetes mit Symptomen wie Polyurie, Polydipsie usw. als Beginn der Erkrankung galt, wird heute in der internationalen Leitlinie und von führenden Wissenschaftlern die Phase davor, nämlich der Nachweis von 2 oder mehr Autoantikörpern, als «Stadium 1» der Erkrankung betrachtet. Trotz vieler Neuerungen bei der Insulintherapie - man denke nur an Insulinpumpen, kontinuierliche Messgeräte, Apps - gelingt es leider weiterhin bei einem gro-
Ben Teil der Patienten nicht, eine optimale Stoffwechselkontrolle zu erreichen, und viele Patienten sind im Laufe ihres Lebens von Folgeerkrankungen betroffen.

Was kann aber getan werden, um die Prognose für Menschen mit Typ-1-Diabetes zu verbessern und/oder bereits in einer frühen Phase einzugreifen, und wie kann man betroffene Menschen möglichst früh identifizieren? Hierzu möchte ich Ihnen unbedingt die Lektüre des Reviews von Sandra Lord und Carla Greenbaum in dieser Ausgabe empfehlen. Interessant ist der Einsatz von Immuntherapeutika wie Teplizumab, einem monoklonalen humanisierten AntiCD3-Antikörper. In einer im NEW ENGLAND JOURNAL OF MEDICINE publizierten Studie konnte gezeigt werden, dass die Anwendung von Teplizumab in einer sehr frühen Phase des Diabetes den tatsächlichen klinischen «Ausbruch» der Erkrankung gegenüber einer Placebo-Gruppe im Median um 24 Monate verzögern konnte. Die kürzlich in SCIence Translational Medicine publizierte Nachuntersuchung zeigt, dass bei 50\% der behandelten Patienten auch nach knapp 3 Jahren noch kein klinisch manifester Diabetes besteht, im Vergleich zu 22\% aus der Pla- 
cebo-Gruppe. Aktuell wird der Nutzen des Medikaments in einer groß angelegten internationalen Studie bei Kindern und Jugendlichen in einer etwas späteren Phase der Erkrankung untersucht («Protect-Studie»).

Darüber hinaus werden in dem Artikel Möglichkeiten der Früherkennung besprochen. So nahmen an der «Fr1da-Studie», einem bayernweiten Pilotprojekt, bei dem Kinder aus der Allgemeinbevölkerung hinsichtlich des Auftretens von Autoantikörpern untersucht wurden, bereits weit mehr als 100000 Kinder teil. Die Prävalenz von Ketoazidosen ist bei Teilnehmern aus der Studie, die einen klinisch manifesten Diabetes entwickeln, sehr gering $(<5 \%)$. Darüber hinaus hat sich die europaweite Plattform GPPAD (Globale Plattform zur Prävention des Autoimmunen Diabetes) zum Ziel ge- setzt, eine internationale Infrastruktur für Studien zur Vorbeugung der Entstehung von Typ-1-Diabetes zu etablieren. Aktuell werden hier unter anderem innovative Therapien für sehr junge Kinder mit genetischem Risiko für Typ-1-Diabetes untersucht. Erfolgversprechende Ansätze arbeiten mit oralem Insulin (POInT-Studie) bzw. Bifidobakterien (SINT1A-Studie). Früherkennung und Immunintervention haben ein hohes Potenzial, die Prognose von Patienten mit Typ-1-Diabetes zu verbessern.

Sehr interessant ist auch der Case Report in dieser Ausgabe von Kompass AutOIMmun: Eindrucksvoll wird anhand von Patientenfällen gezeigt, dass starke Einschränkungen in der Ernährung, wie z.B. eine Restriktion von Kohlenhydraten, bei Kindern und Jugendlichen nicht zu empfehlen sind. Auch wenn derartige Diäten möglicherweise pri- mär zu einer Verringerung von Glukoseexkursionen führen, so zeigen sich doch negative Folgen wie Wachstums- oder Gedeihstörungen.

Weitere Artikel rund um die Themen Typ1-Diabetes, Komplikationen, neue Therapien, aber natürlich auch zu anderen Autoimmunerkrankungen, finden Sie in diesem Heft. Lohnend ist auch der «Steckbrief Forschung», in dem eine junge Forscherin auf dem Gebiet des Diabetes vorgestellt wird. Ihnen allen wünsche ich viel Vergnügen bei der Lektüre dieser sehr spannenden Ausgabe von Kompass AUTOIMMUN. Ihre

\section{Katharina Waruche}

PD Dr. Katharina Warncke 